\title{
All that glitters is not gold
}

\author{
R P Mookerjee, R Jalan
}

\section{Addition of vasoactive drug therapy improved the effectiveness of injection sclerosis or band ligation for acute variceal bleeding but failed to produce a significant reduction in mortality}

in nitial presentation with variceal bleeding is associated with a $20-30 \%$ risk of mortality within the first two weeks. Early control of bleeding is paramount and traditionally has taken the form of endoscopic therapy to mechanically reduce variceal blood flow, or vasoactive drugs that decrease splanchnic and/or azygous blood flow. Although sclerotherapy has been shown to reduce bleeding compared with placebo, ${ }^{1}$ it results in more frequent adverse events than medical interventions ${ }^{2}$ and has largely been superseded by variceal band ligation. Indeed ligation has been shown to reduce rebleeding and mortality compared with sclerotherapy and is the current gold standard for acute bleeding and secondary prophylaxis. ${ }^{3}$ A recent evaluation combining the two endoscopic modalities demonstrated no benefit over ligation alone in preventing rebleeding or mortality. ${ }^{4}$

The systematic review by Banares et al suggests a potential benefit from combining vasoactive drugs with endoscopic therapy with improved initial endoscopic control of bleeding and five day haemostasis, albeit with no reduction in mortality. This is a high quality systematic review with assessment of the quality of all included trials and sensitivity analysis to establish any significant heterogeneity between trials. Exclusion criteria have been well defined and attention given to publication bias and differences in disease severity between the incorporated trials. However, there are several aspects of this review we wish to highlight as being important in judging whether there is a justification for using somatostatin analogues as adjuvants to endoscopic therapy in the management of acute variceal haemorrhage: (1) The value of data on five day haemostasis is difficult to interpret from a pathophysiological perspective as it is not equivalent to established control of bleeding during the "at risk" period which is the duration of admission. In other words, we feel that this outcome is somewhat artificial.

(2) In only two of the eight trials included was variceal ligation the endoscopic therapy used and thus the trials do not represent current clinical practice where ligation is the standard intervention.

(3) Despite 939 patients being reviewed from eight trials, there was no statistically significant survival benefit from

Banares R, Albillos A, Rincon D, et al. Endoscopic treatment versus endoscopic plus pharmacologic treatment for acute variceal bleeding: a meta-analysis. Hepatology 2002;35:609-15.

Background: While endoscopic therapy involving either injection sclerosis or band ligation is considered the intervention of first choice for acute variceal bleeding, pharmacological agents have also been shown to be highly effective in the control of bleeding. Even so, acute variceal bleeding continues to carry a substantial mortality making the combination of endoscopic therapy and vasoactive drug therapy a logical approach.

Question: Does the addition of vasoactive drug therapy improve the effectiveness of injection sclerosis or band ligation for acute variceal bleeding? Methods: Systematic review and meta analysis of randomised control trials published between 1994 and 2001, identified from a comprehensive search, including electronic databases, the Cochrane registry of trials, and abstracts from major meetings.

Results: Eight trials (three only published as abstracts) involving 939 patients $161 \% \mathrm{men})$ fulfilled the selection criteria. Alcoholic cirrhosis was present in $66 \%$, and $39 \%$ of patients were Child class C. Combined treatment improved initial control of bleeding (relative risk (RR) 1.12; 95\% confidence interval 1.02-1.23) and also improved haemostasis assessed at five days (RR 1.28; 1.8-1.39), with the numbers of patients needed to treat to avoid one patient with further or repeated bleeding being 8 and 5 , respectively. These benefits of combined treatment remained significant when trials used drugs other than octreotide or that included a low proportion of patients with alcohol induced cirrhosis $(<40 \%)$ or high risk cirrhotic patients $(<35 \%)$ were excluded. Despite these improvements, mortality was not significantly decreased by combined therapy (RR 0.73; 0.451.18). Severe adverse events were similar in both groups.

Conclusion: In patients with acute variceal bleeding, a vasoactive drug therapy improved both initial and five day control of bleeding but failed to produce a significant reduction in mortality.

combined treatment, raising the question as to whether the rationale for using this expensive intervention with limited benefits is justifiable. Although the five trials published in peer review journals suggest a reduced transfusion requirement, there are few data on the cost implications of intensive care requirements, duration of hospital stay, or additional interventions required, except for the trial by Sung et al which included treatment with variceal ligation and demonstrated reduced requirements for balloon tamponade. $^{5}$

(4) The authors amalgamated data on somatostatin, octreotide, and vapreotide making the benefit of a particular agent more difficult to interpret. Furthermore, octreotide as an adjunct to treatment is difficult to assess as the dose required, mode of administration, and variability of response are much debated, stemming from the documented rapid desensitisation to its effects. ${ }^{6}$ The main effect of octreotide relates to a reduction in the postprandial rise in portal pressure. By comparison, terlipressin is a vasoactive treatment with proven mortality benefit from bleeding varices compared with placebo, ${ }^{7}$ has fewer adverse events, and is as efficacious as sclerotherapy. ${ }^{8}$ The authors chose to exclude a terlipressin treatment arm in one of the included studies $^{9}$ and it is possible that inclusion of data from trials with terlipressin may have demonstrated a favourable mortality benefit.

(5) A further limitation of the trials included is the very limited data on adverse events which were reported in only three of the eight trials. Defining the adverse event profile of combined therapy is clearly vital if this is to be incorporated into future management guidelines.

Current data are not sufficient to justify the use of vasoactive drugs as an adjuvant to endoscopic therapy. Clearly, larger trials comparing variceal band 
ligation alone with ligation plus vasoactive drug therapy, with inhospital mortality as the primary end point, are needed to determine the exact place of these agents as adjuvants to endoscopic therapy.

Gut 2002;51:623-624

\section{..................}

\section{Authors' affiliations}

R P Mookeriee, R Jalan, Liver Failure Group, Institute of Hepatology, University College London, London, UK

Correspondence to: Dr R Jalan, Liver Failure Group, Institute of Hepatology, University College London, 69-75 Chenies Mews, London WCIE 6HX, UK; r.jalan@ucl.ac.uk

\section{REFERENCES}

1 MacDougall BR, Westaby D, Theodossi A, et al. Increased long-term survival in variceal haemorrhage using injection sclerotherapy. Results of a controlled trial. Lancet 1982;1:124-7.

2 D'Amico G, Pietrosi G, Tarantino l, et al. Emergency sclerotherapy versus medical interventions for bleeding oesophageal varices in cirrhotic patients. Cochrane Database Syst Rev 2002:CD002233.

3 Laine L, el-Newihi HM, Migikovsky B, et al. Endoscopic ligation compared with sclerotherapy for the treatment of bleeding esophageal varices. Ann Intern Med 1993; 119:1-7.

4 Singh $\mathbf{P}$, Pooran $N$, Indaram $A$, et al. Combined ligation and sclerotherapy versus ligation alone for secondary prophylaxis of esophageal variceal bleeding: a meta-analysis. Am J Gastroenterol 2002;97:623-9.
5 Sung JJ, Chung SC, Yung MY, et al. Prospective randomised study of effect of octreotide on rebleeding from oesophagea varices after endoscopic ligation. Lancet 1995;346: 1666-9.

6 Escorsell A, Bandi JC, Andreu V, et al. Desensitization to the effects of intravenous octreotide in cirrhotic patients with portal hypertension. Gastroenterology 2001:120:161-9.

7 loannou G, Doust J, Rockey DC. Terlipressin for acute esophageal variceal hemorrhage. Cochrane Database Syst Rev 2001:CD002147.

8 Escorsell A, Ruiz del Arbol L, Planas R, et al. Multicenter randomized controlled trial of terlipressin versus sclerotherapy in the
treatment of acute variceal bleeding: the TEST treatment of acute variceal bleeding:

9 Ceriani R, Curioni R, Morini L, et al.

Ceriani R, Curioni R, Morini L, et al.
Sclerotherapy alone (ES) vs. sclerotherap plus terlipressin (EST) vs. sclerotherapy plus octreotide (ESO) in the treatment of acute variceal bleeding. Gastroenterology 